7 Noetzel H. Diffusion von Blutfarb in der inneren Randzone und aussere Oberflache des Zentralnervensystems bei subarachnoidaler Blutung. Arch Psychiat Nervenkr 1940;111:129-38.

8 Trevor Hughes J, Oppenheimer DR. Superficial siderosis of the central nervous system. Acta Neuropathol (Berl) 1969;13:56-74.

9 Bradley WG. Diseases of the spinal roots. In: Dyck PJ, Thomas PK, Lambert EH, Bunge R, eds. Peripheral Neuropathy. Philadelphia: Saunders, 1984:1377.

10 Adams RD, Victor M. Principles of Neurology. New York: McGraw-Hill 1985:472.

Accepted 4 August 1987

\section{Complete gaze palsy in pontine haemorrhage}

Sir: Henn et $a l^{1}$ have recently shown that in monkeys lesions confined to the reticular formation of the pons (paramedian pontine reticular formation, PPRF) cause abnormalities of vertical as well as horizontal gaze. In man it has long been recognised that pontine lesions cause horizontal gaze defects but vertical palsies have rarely been recorded. We report a patient with a pontine haemorrhage in whom there was both horizontal and vertical gaze palsy.

A 55 year old hypertensive male was admitted to hospital with sudden onset of severe walking difficulty and left sided paraesthesia. On examination his blood pressure was $230 / 140 \mathrm{~mm} \mathrm{Hg}$. He was co-operative and alert. Visual acuity and clinical visual field testing were normal. His fundi showed Grade II retinopathy. There was bilateral symmetrical miosis, but the pupils reacted to light and the eyelids were normally posi- tioned. The patient's eyes were in the primary position and he could not move them on command or in response to stationary or moving visual targets. Doll's head manoeuvre in the horizontal plane, (vestibulo-ocular reflex), as well as caloric irrigation with water at about $20^{\circ} \mathrm{C}$ failed to induce any eye movement. There was a right sided lower motor neuron facial weakness. The patient was unable to walk because of severe gait ataxia but sensorimotor examination of the limbs while lying down was normal. Tendon reflexes and plantar responses were also normal.

Investigations included normal CSF examination. A CT scan (fig a) showed a pontine haemorrhage occupying the medial tegmental area, slightly more on the right, extending up to the ponto-mesencephalic junction. Mesencephalic and mesencephalon-diencephalic junction sections (fig b) were normal. The patient's hypertension was controlled with propranolol (160 mg a day) and nifedipine (40 $\mathrm{mg}$ a day). On the third day the patient noticed diplopia and oscillopsia and examination showed he had recovered a few degrees of conjugate vertical eye movements and abduction of the left eye, accompanied by left beating nystagmus on attempted gaze to that side. After a week the patient's gait improved and he was able to walk with help although he suffered from vertigo on standing. During the second week vertical gaze and convergence were restored. Abduction in the left eye was partially recovered. There was a conjugate, torsional nystagmus to the left (clockwise) and a superimposed horizontal pendular nystagmus in the left eye. By the eighth week the haematoma had resolved on CT scanning.
When examined 2 years later the patie had mild cerebellar signs in the left limbs and an unsteady, broad based gait with leat lateropulsion. He complained of position vertigo and horizontal oscillopsia which w\&8 worse when viewing with the left eye alons There was a horizontal pendular nystagmue with an elliptical trajectory more marked of the left eye, a constant clockwise torsiona nystagmus in both eyes, a severe right nerve palsy, bilateral horizontal gaze palsy much worse towards the right, and a right internuclear ophthalmoplegia (one and half syndrome); these horizontal gaze defects were less marked on pursuit anf doll's head manoeuvre. The rest of the e窟 movements were normal.

Henn and co-workers showed tha restricted caudal lesions in the PPRF cact produce permanent abolition of horizontal and vertical saccades and quick componens of nystagmus in the monkey. ${ }^{1}$ Clinicat examples of documented central pontinf lesions in cooperative patients are not ves common and the effects on vertical ga have been variable and contradictory. ${ }^{1-8}$ For instance, no case reported had a pert manent vertical gaze palsy; some of the patients showed normal vertical eye mover ments whereas others had only slowed vert? cal saccades. The case reported here frad clinical and radiological evidence of a tine lesion. However, the lesion was restricted to the PPRF, since smooth gue suit, saccades and the vestibulo-ocular refle were also affected. It is possible that involvement of other neighbouring pofin structures such as the VI nuclei, ML $\mathscr{E}^{\circ}$ and/or vestibular pathways may partly account for this.

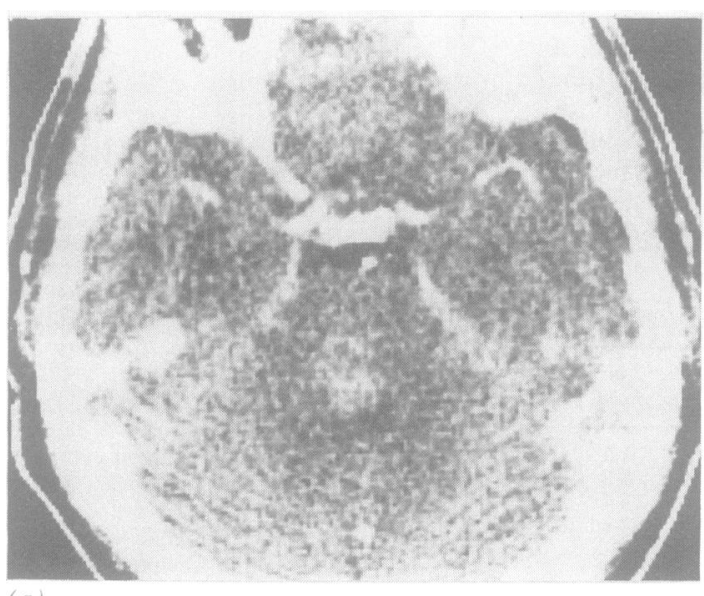

Fig (a) Enhanced CT scan showing tegmental haemorrhage in the pons. (b) Normal appearance of the enhanced CT scan at the level of the superior colliculi.

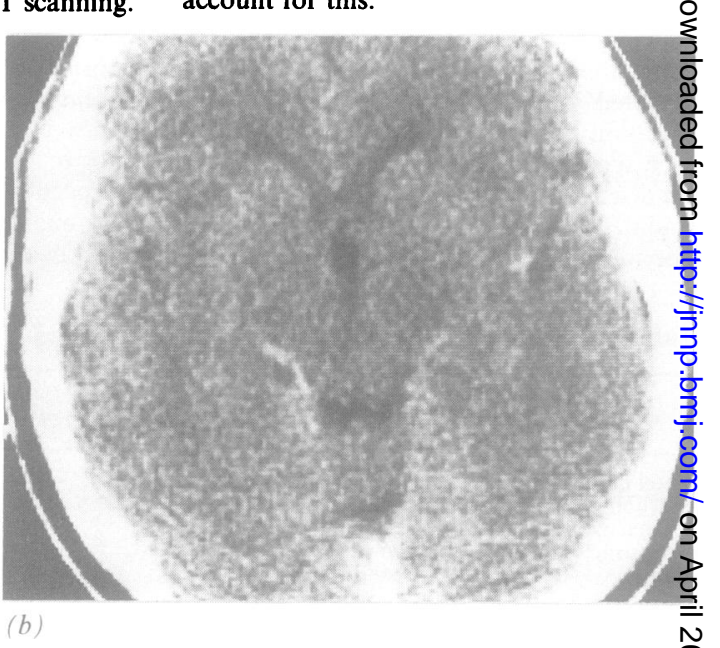


The rostral interstitial nucleus of the MLF (ri MLF) is thought to be the immediate pre-nuclear structure for generating vertical saccades and receives an ascending input from the PPRF. ${ }^{1}$ This nucleus, located in the rostral mesencephalic reticular formation, was almost certainly spared in our case since there was no clinical or radiological evidence of damage to that region; consciousness was never impaired and, although there was initially impairment of convergence, the absence of mydriasis, ptosis and vertical strabismus exclude significant III nerve nuclear or infranuclear involvement. The most likely explanation for the transient vertical gaze palsy in our patient is damage of the PPRF. However, if the PPRF were crucial for the generation of vertical eye movements in man, as it is in the monkey, an explanation is needed for the paucity of cases in the literature. Descending fibres from the frontal eye fields terminate in the mesencephalic and pontine reticular nuclei related to ocular-motor control. ${ }^{7}$ Thus, it might be postulated that the ri MLF can be activated from two sources: the first and normal source is the PPRF. Such a source enables integration to occur between the horizontal and vertical vector of any eye movements and is necessary for the development of oblique saccades which require simultaneous discharge from both mesencephalic and pontine reticular gaze centres. If the PPRF is damaged a second source is the direct descending pathway to the mesencephalic gaze centres, which could be reweighted such that vertical saccades can again be generated. Although this is entirely speculative is is a possible explanation of the variability and transience of vertical gaze defects in pontine lesions. In this or any other explanation, however, the underlying assumption is that there may be differences in the control of vertical saccades between man and monkey.

We are grateful to the Sociedad Neurologica Argentina and Laboratorios Janssen, Argentina, for travelling support, Dr J Sierra, who carried out the CT scans in FLENI, Buenos Aires, and Dr Peter Rudge who kindly reviewed the manuscript.

RO DOMINGUEZ Servicio de Neurologia, Hospital Sirio-Libanes, Buenos Aires, Argentina AM BRONSTEIN

Medical Research Council Neuro-Otology Unit, Institute of Neurology, National Hospital, Queen Square, London, UK
Address for correspondence: Dr AM Bronstein MRC Neuro-Otology Unit, National Hospital, Queen Square, London, WCIN $3 B G, U K$

\section{References}

1 Henn V. Lang W, Hepp K, Reisine H. Experimental gaze palsies in monkeys and their relation to human pathology. Brain 1984; 107:619-36.

2 Hennerici M, Fromm C. Isolated complete gaze palsy: an unusual ocular movement deficit probably due to a bilateral parapontine reticular formation (PPRF) lesion. Neuroophthalmol 1981:1:165-73.

3 Larmande P, Henin D, Jan M, Gouaze A. Abnormal vertical eye movements in the locked-in syndrome. Ann Neurol 1982:11:100-2.

4 Pierrot-Deseilligny C, Goasguen J, Chain F Lapresle J. Pontire metastasis with dissociated bilateral horizontal gaze paralysis. J Neurol, Neurosurg, Psychiatry 1984;47: 159-64.

5 Baloh RW, Furman J, Yee RD. Eye movements in patients with absent voluntary horizontal gaze. Ann Neurol 1985:17:283-6.

6 Hanson MR. Hamid MA, Romsak RL, Chou SS. Leigh RJ. Selective saccadic palsy caused by pontine lesions: Clinical, Physiological, and Pathological Correlations. Ann Neurol 1985:20:209-17

7 Leichnetz GR. The prefrontal corticooculomotor trajectories in the monkey. $J$ Neurol Sci 1981:49:367-96.

Accepted 14 July 1987

Late onset posthemiplegic dystonia in systemic lupus erythematosus

Sir: Delayed development of focal dystonic or athetoid movements following hemiplegia (late onset posthemiplegic dystonia) is rare but well documented. ${ }^{1-6}$ We report a case of late onset posthemiplegic dystonia in a patient with systemic lupus erythematosus (SLE). To our knowledge this association has not been previously reported.

At age 42 years, a female patient developed a left hemiparesis that subsequently resolved completely. A year later she was hospitalised in our Neurology Department because of right hemiparesis and speech disturbances. CT of the head demonstrated the presence of two hypodense nonenhancing lesions, one in the left basal ganglia and internal capsule area end a smaller one in the right lentiform nucleus. Sedimentation rate was $90 \mathrm{~mm} / \mathrm{h}$ and ANF positive with a titre 1:80. The rest of the laboratory tests. including extensive investigation for collagen vascular disease, were negative. Two years later she noticed abnormal movements involving the left sided extremities, which progressed to the point that she lost the ability to use her left hand and had difficulty in walking.

Neurological examination on admission revealed a low output speech with no other aphasic features and a right spastic hemiparesis. The left arm was held in adduction at the shoulder and flexion at the elbow, while the hand was in extension and ulnar deviation at the wrist. The fingers were in extension at the carpometacarpal joints and exhibited prolonged, sustained twisting movements, which increased during attempted use of the arm. The left leg was in extension at the knee, while the foot was in inversion and plantar extension. Deep tendon reflexes were brisk bilaterally but more so on the right. An extensor plantar response was elicited on the right. No sensory abnormalities were found

A repeat CT scan of the head again demonstrated two hypodense lesions in the same areas of the basal ganglia. Other abnormal laboratory data included: sedimentation rate $135 \mathrm{~mm} / \mathrm{h}$, red cell count $2,600,000 \mathrm{~mm}^{3}$. white cell count $3,000 \mathrm{~mm}^{3}$ with a differential count of $62 \%$ neutrophils, $28 \%$ lymphocytes, $4 \%$ eosinophils and $6 \%$ monocytes; platelet count $30.000 \mathrm{~mm}^{3}$ BUN was $4.32 \mathrm{mmol}$ urea $/$, creatinine 114.9 umol/1. ANF positive $(1: 2560)$, anti-DNA positive, anti-ENA positive. Total serum complement was $16.2 \mathrm{kU} / \mathrm{ml}$ (normal: 35-45) with C3 9.2 SIU (normal: 100-250) and C4 21 SIU (normal: 20-65). VDRL and FTA were negative. LE cells were present.

The patient was subsequently treated with steroids. The blood picture improved but the neurological manifestations remained unchanged. Two months after her discharge she developed seizures

In the present case the neurological symptoms preceded other clinical manifestations of SLE by 4 years. In the few previously reported cases choreoathetoid movements in SLE have had a rather acute onset and later subsided. ${ }^{7}$ However, in our patient the abnormal movements developed slowly and progressively and involved the left sided extremities only after the weakness had subsided. The movements included sustained athetoid twisting of the fingers and dystonic posturing of the more proximal parts of the involved extremities, which resembled those described in other cases of post-hemiplegic dystonia. $^{1-6}$

We consider that the low density lesion in the right putamen. apparently an infarct, 